\title{
VDAC/HK2 Modulator Ointment VDA-110
}

National Cancer Institute

\section{Source}

National Cancer Institute. VDAC/HK2 Modulator Ointment VDA-110. NCI Thesaurus.

Code C131826.

A topical ointment formulation containing a voltage-dependent anion channel (VDAC)/hexokinase 2 (HK2) modulator, with potential activity ag ainst certain skin cancers. Upon topical application, VDA-1102, as a protein-protein interaction (PPI) disrupter, allosterically binds to HK2, and causes HK2 to dissociate from VDAC, which prevents glycolysis and triggers apoptosis, thereby inhibiting tumor cell growth in VDAC/HK2-expressing tumor cells without affecting the surrounding healthy skin tissue. The VDAC/HK2 system, over-activated in cancer cells, plays a key role in tumor cell proliferation. The VDAC/HK2 association protects cancer cells from apoptosis. HK2 is overexpressed in many malignant cancer tissues that rely on glycolysis, while its expression is limited in normal healthy cells. 